\title{
Method for fast computation of angular light scattering spectra from 2D periodic arrays
}

\author{
Jan Pomplun, ${ }^{a}$ Sven Burger, ${ }^{a b}$ Lin Zschiedrich, ${ }^{a}$ Philipp Gutsche, ${ }^{a b}$ Frank Schmidt ${ }^{a b}$ \\ ${ }^{a}$ JCMwave GmbH, Bolivarallee 22, D - 14050 Berlin, Germany \\ ${ }^{b}$ Zuse Institute Berlin (ZIB), Takustraße 7, D-14195 Berlin, Germany
}

\begin{abstract}
This paper will be published in Proc. SPIE Vol. 9778 (2016) 977839 (Metrology, Inspection, and Process Control for Microlithography XXX, DOI: 10.1117/12.2219666) and is made available as an electronic preprint with permission of SPIE. One print or electronic copy may be made for personal use only. Systematic or multiple reproduction, distribution to multiple locations via electronic or other means, duplication of any material in this paper for a fee or for commercial purposes, or modification of the content of the paper are prohibited. Please see original paper for images at higher resolution.
\end{abstract}

\begin{abstract}
An efficient numerical method for computing angle-resolved light scattering off periodic arrays is presented. The method combines finite-element discretization with a Schur complement solver. A significant speed-up of the computations in comparison to standard finite-element method computations is observed.
\end{abstract}

Keywords: 3D electromagnetic field simulations, finite-element method, Schur-complement, scatterometry, optical metrology, computational lithography

\section{INTRODUCTION}

Optical metrology is used in semiconductor and photomask manufacturing processes to determine a variety of characteristics of patterned wafers or photomasks such as layer thicknesses, width and shape of patterned structures, material properties and distribution, or overlay between different layers or structures. State-ofthe-art methods determining these features include spectroscopic or angle-resolved scatterometry, ellipsometry, reflectometry or imaging based methods ${ }^{1}$ These methods consist of an optical system which illuminates the measured sample and a detector system which measures properties of the light after interaction with the sample, e.g., the intensity, phase and/or polarization state of reflected and/or transmitted, resp. of scattered light. For interpretation of the measurement, the measurement data are related to results obtained from modeling and simulation of the measurement setup for different values of the sample characteristics. These modeled distributions can be computed during the measurement process or can be precomputed in advance and stored in libraries. For a large number of free parameters, computation of these modeled light distributions can be very demanding. Free parameters are physical characteristics of the modeled sample, such as geometrical or material properties. Further, a number of parameters is needed to describe the illumination system, e.g., multiple wavelengths and incidence directions or shape and polarization of the light beam. The incident light field generated by the illumination system is typically a beam with a finite numerical aperture, incident from a specific direction, or it has a complex shape, e.g., in quadrupole or freeform illumination. Such light distributions are typically modeled by a superposition of a number of fields, e.g., plane waves, with different incident directions, phases, and intensities.2 hundreds of fields).

If the measured sample is modeled as a periodic pattern, rigorous computation methods like the Finite Element Method ${ }^{3}$ (FEM), Rigorously Coupled Wave Analysis (RCWA) or Finite Difference Time Domain Method

Corresponding author: S. Burger

URL: http://www.jcmwave.com

URL: http://www.zib.de 
(FDTD) generally have to compute a solution separately for each incident direction. In addition to the variable physical characteristics, this significantly increases time and effort to generate the necessary modeled light distributions. The case of periodic patterns is highly important in semiconductor manufacturing and other technologies since many fabricated structures exhibit periodicity. Similar requirements for computations of solutions to Maxwell's equations as in the above described field of computational metrology are present, e.g., in the field of computational lithography

A need therefore exists for a method to speed-up computation of such light distributions incident from a number of different directions on periodic samples. In this contribution we present results from an implementation of a Schur complement based method $\sqrt{5}$ allowing for such speed-ups. We demonstrate results that are based on JCMsuite, a FEM solver for the linear Maxwell's equations in frequency domain. The implementation contains features like higher-order edge-elements, ${ }^{6}$ domain-decomposition, $\left.{ }^{7}\right]$ hp-adaptivity, ${ }^{8}$ and model order reduction ${ }^{9}$ It is applied to tasks in computational lithography and metrology 13 as well as to further fields in technology and research, see, e.g., for an overview 14

This paper is structured as follows: The background of the numerical method is presented in Section 2 Section 3 presents simulation results on computation of angle resolved reflection spectra from 2D-periodic arrays of silicon nano-posts.

\section{SCHUR COMPLEMENT METHOD}

In this contribution we present a method to efficiently perform computation of scattered light fields from a periodic sample for a number of incident directions. The computational effort scales only very slowly with the number of incident directions. This can lead to substantial savings up to orders of magnitude in time and hardware costs for the computer system of a metrology or computational lithography system.

The general idea of the presented method is the decomposition of the light scattering model into a part which is independent on the direction of the incident field and a part which depends on the direction of the incident field. The part which is independent on the incident direction is solved once for all directions. The part dependent on the incident direction is solved for each incident direction individually. The individual and the common solutions are combined to give the total solution.

As a prerequisite let us look at a linear system of equations (as resulting, e.g., from FEM discretization of Maxwell's equations ${ }^{6}$ ):

$$
A x=f,
$$

where $A$ is a matrix, $f$ is the right hand side, and $x$ is the sought solution.

Now we split the matrix into following blocks:

$$
A=\left(\begin{array}{ll}
A_{11} & A_{12} \\
A_{21} & A_{22}
\end{array}\right)
$$

where $A_{11}$ and $A_{22}$ are square matrices of generally different dimensions. Further, we split the solution and right hand side into corresponding blocks:

$$
\begin{aligned}
& x=\left(\begin{array}{l}
x_{1} \\
x_{2}
\end{array}\right), \\
& f=\left(\begin{array}{l}
f_{1} \\
f_{2}
\end{array}\right) .
\end{aligned}
$$

Using this decomposition, Eq. (1) can be written as follows:

$$
\begin{aligned}
& A_{11} x_{1}+A_{12} x_{2}=f_{1} \\
& A_{21} x_{1}+A_{22} x_{2}=f_{2} .
\end{aligned}
$$


Equation (5) can be solved with respect to $x_{1}$ :

$$
x_{1}=A_{11}^{-1}\left(f_{1}-A_{12} x_{2}\right)
$$

and used in (6), giving:

$$
\left(A_{22}-A_{21} A_{11}^{-1} A_{12}\right) x_{2}=f_{2}-A_{21} A_{11}^{-1} f_{1} .
$$

Matrix $\left(A_{22}-A_{21} A_{11}^{-1} A_{12}\right)$ is the so-called Schur complement ${ }^{5}$ of $A$.

Now let us consider typical light scattering models, arising from discretization of Maxwell's equations, using e.g., FEM, FDTD, Discontinuous Galerkin, Finite Volume Method, or Volume Integral Method. The scattering model from a periodic structure is thereby formulated on a single unit cell and periodic boundary conditions are applied. These periodic boundary conditions depend on the grid vectors of the periodic domain and the incidence direction of the incoming light field, i.e., the phase difference from opposite periodic boundaries. This means that a number of entries in matrix $A$ depend on the incident direction and hence have to be solved independently.

In order to use above Schur splitting we reorder the linear system, such that all entries independent on the incident direction $\theta$ are in block $A_{11}$ :

$$
\begin{aligned}
A_{11} x_{1}+A_{12}(\theta) x_{2} & =f_{1}(\theta) \\
A_{21}(\theta) x_{1}+A_{22}(\theta) x_{2} & =f_{2}(\theta) .
\end{aligned}
$$

Hence, looking at (7) and (8) we observe that inversion of block $A_{11}$ has to be performed only once, independent on the incidence direction. Only the solution of (8) is needed for each incidence direction.

Typically the size of $A_{11}$ is much larger than the size of $A_{22}$ since the size of block $A_{22}$ only corresponds to the degrees of freedom on the boundary of the periodic computational domain. These are much less than the number of degrees of freedom in the volume in block $A_{11}$.

In order to arrive at the Schur system (8) the inverted (i.e., LU-decomposed) matrix is used for solving following systems:

$$
\begin{gathered}
A_{11}^{-1} A_{12}(\theta) \\
A_{11}^{-1} f_{1}(\theta),
\end{gathered}
$$

and the solutions are used in (8) to assemble the $\theta$ dependent Schur complement:

$$
A_{22}(\theta)-A_{21}(\theta) A_{11}^{-1} A_{12}(\theta)
$$
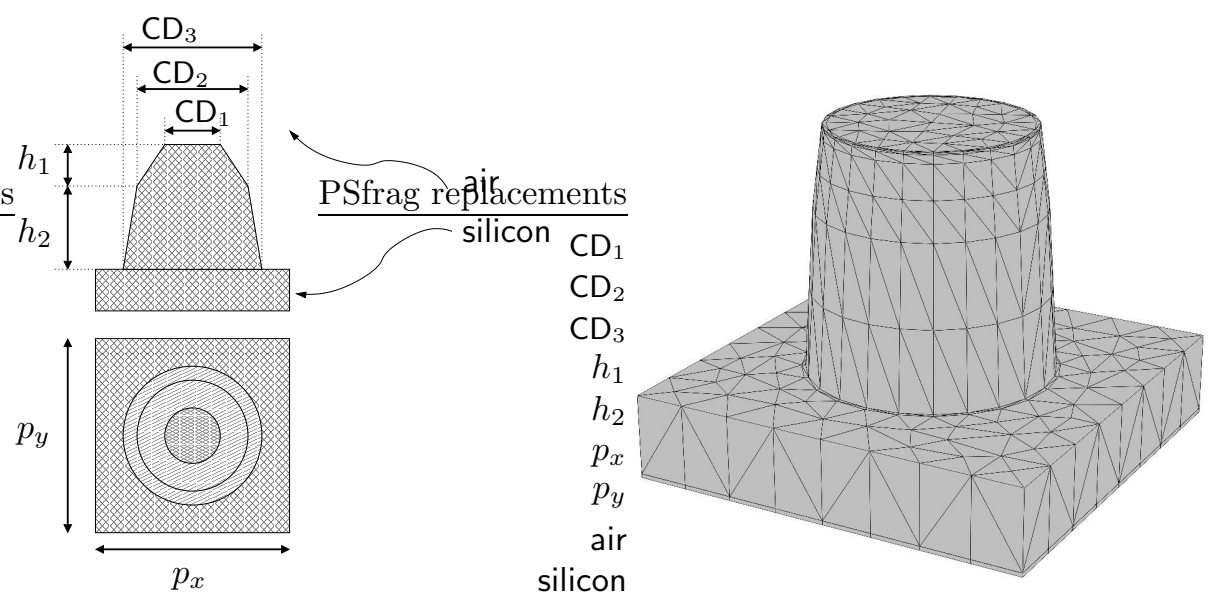

Figure 1. Left: Schematics of the geometry of the investigated scattering geometry (unit cell of a 2D-periodic grating of nano-rods with circular cross section. Parameters of the model are indicated (critical dimension, CD, at different heights, $h_{1}, h_{2}$, pitches $\left.p_{x}, p_{y}\right)$, cf., Table1 Right: Visualization of the mesh discretizing the geometry (only silicon parts shown). 
Once this dense Schur system is solved for $x_{2}$, the inner solution $x_{1}$ can be obtained from (7). Also here, the decomposition of $A_{11}$ can be used for all incidence directions. An increasing number of incidence directions only leads to an increasing number of right hand sides. Especially for discretization of 3D scattering problems, however the LU-decomposition of the system takes much more effort than solving for a large number of right hand sides.

In the following example we will show that the Schur splitting can lead to substantial savings in computation time when scattering problems with a large number of right hand sides (i.e., sources with various incidence directions) are solved.

\section{APPLICATION EXAMPLE: LIGHT SCATTERING OFF AN ARRAY OF NANO-POSTS}

In this section, the computation of angle-resolved scattering spectra is analyzed as an example of the described Schur complement technique. Corresponding setups are relevant for applications like, e.g., Fourier ellipsometry 13 The following model is investigated: Light with a vacuum wavelength of $\lambda_{0}=300 \mathrm{~nm}$ and well-defined polarizations (S- and P-polarized) illuminates a 3D structure. The source is modeled as a series of independent, coherent plane waves with well defined wave-vectors $\vec{k}=\left(k_{x}, k_{y}, k_{z}\right)$. For normal incidence, the sample is arranged such that $\vec{k}=\left(0,0, k_{z}\right), k_{z}=2 \pi / \lambda_{0}$. The geometry of the illuminated structure is a $2 \mathrm{D}$-periodic array of nano-rods. The material corresponds to silicon, with a complex refractive index $n=5.0+4.16 i$ at the given wavelength obtained from tabulated data. 15 The superspace is modeled as air, with refractive index $n=1.0$. The parameterized geometry is schematically shown in Fig. 1, and the chosen geometrical parameter set is as defined in Table 1.

\begin{tabular}{|l|l|}
\hline$p_{x}=p_{y}$ & $100 \mathrm{~nm}$ \\
\hline $\mathrm{CD}_{1} / \mathrm{CD}_{2} / \mathrm{CD}_{3}$ & $50 \mathrm{~nm} / 55 \mathrm{~nm} / 60 \mathrm{~nm}$ \\
\hline$h_{1} / h_{2}$ & $40 \mathrm{~nm} / 20 \mathrm{~nm}$ \\
\hline
\end{tabular}

Table 1. Parameter settings for the Si nano-post array (compare Fig. 11).

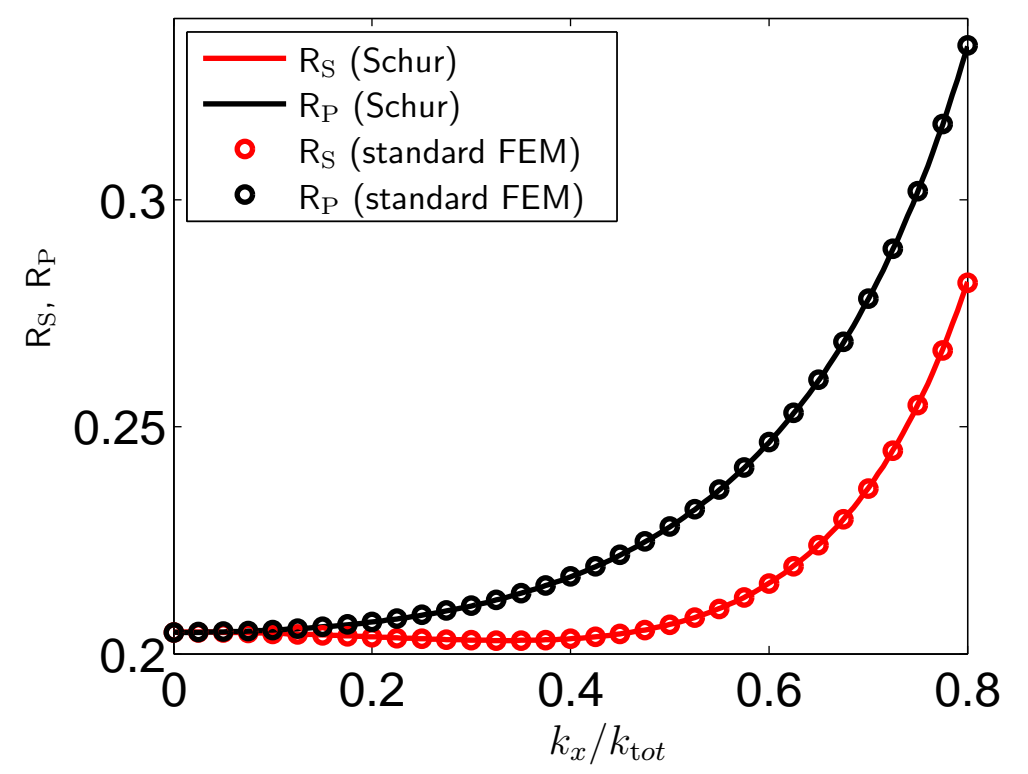

Figure 2. Validation of the Schur complement method by comparison to standard FEM. Angular reflection spectra for $S$ - and P-polarized light incident on the nanorod array, $R_{S}, R_{P}$. The solid lines show results computed with the Schur complement method (for 322 sources). The open circles show results computed with standard FEM for a subset of sources. 
(a)

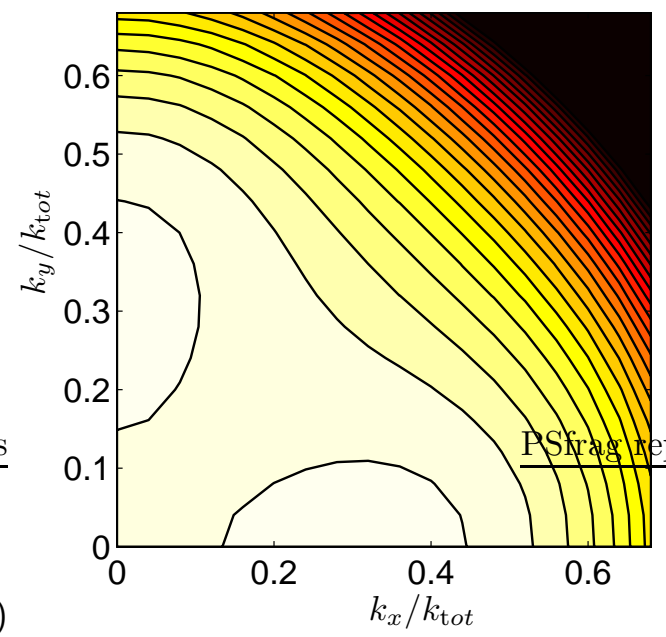

(b)

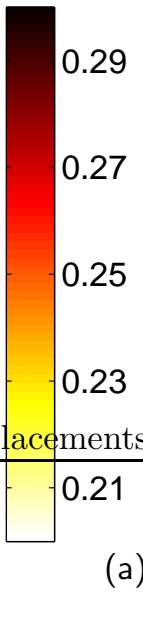

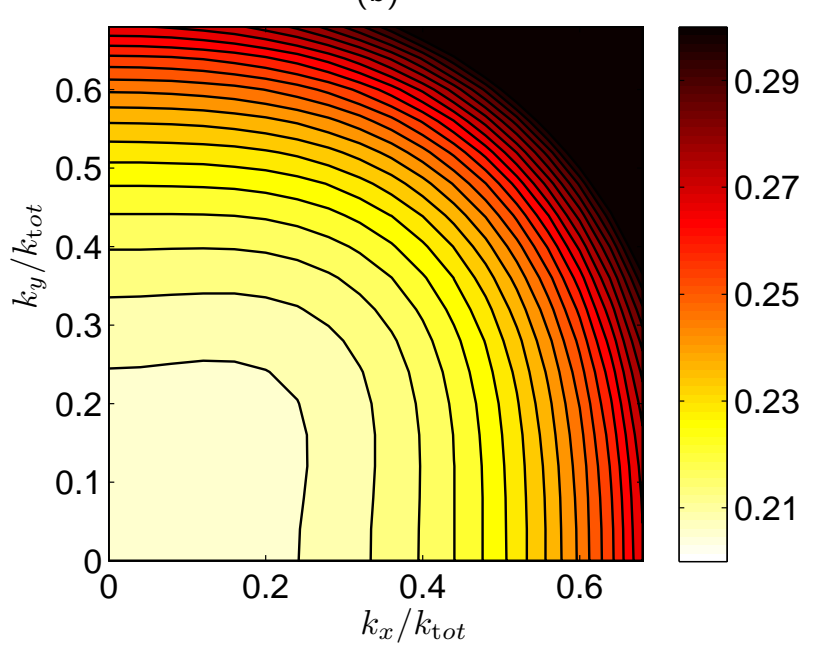

Figure 3. Angle-resolved reflection spectra for S-polarized illumination (a) and P-polarized illumination (b). Both spectra have been obtained in a single simulation run using the Schur complement method for a total of 848 incident source fields.

First, the Schur complement method is validated: Angular reflection spectra of S- and P-polarized light are computed using the Schur complement method, using 161 directions of incidence. Figure 2 shows the obtained spectra. We validate the results by computing the same spectra using standard FEM, i.e., using separate computations for each angle of incidence and not decomposing the matrix $A$ into $\theta$-dependent and $\theta$-independent parts (see Eq. 10). These spectra are also plotted in Figure2 The corresponding results agree quantitatively to a level of a relative error below $0.2 \%$ at the chosen accuracy setting. Here, the precision parameter for the $h p$ refinement of the finite element discretization was chosen as $p_{\mathrm{P} r e c}=4 \times 10^{-3}$, yielding a discrete problem with around $10^{5}$ unknowns. Please see a previous publication for details on the numerical settings for $h p$-refinement $\frac{8}{8}$ Agreement of results obtained with the Schur complement method and with standard FEM is expected, however, small deviations of the numerical results within the ranges of numerical discretization errors are due to differences in numerical treatment of transparent boundaries in the Schur complement and in the standard FEM case.

Next, usability of the method is demonstrated by computing two angle-resolved reflection spectra for the array, where both, the inclination angle and the rotation angle are varied. Figure 3 visualizes the resulting spectra. In the figure, the absolute values of the reflection coefficients for $\mathrm{S}$ and $\mathrm{P}$ polarized light are plotted as function of $k_{x}$ and $k_{y}$, which are the transversal components of the incident wave vector $\vec{k}=\left(k_{x}, k_{y}, k_{z}\right)$. These results have been obtained in a single computation using the Schur complement method, where 848 plane waves with different wave vectors and polarizations have been used. This results demonstrates practicability of the method for simulation projects with many independent source terms.

As commented above, the computational costs of a Schur method simulation run with $N$ sources can be significantly smaller than the total computational cost of $N$ independent simulation runs. To demonstrate this for the given example, the CPU times for computations with both approaches are compared (Schur method CPU times and standard FEM CPU times are normalized with the same constant). Figure 4 shows how the computation times scale with the number of sources. A speed-up by a factor of $>5$ is observed for this specific example. Both simulation runs have been performed with the same numerical accuracy settings (meshing parameters, hp-FEM discretization parameters), and on the same hardware (using a single thread of a standard computer). Please note that for the standard FEM computations we have also "re-used" the inverted system matrix $A^{-1}$ for the second source polarization setting (for each given wave vector). Accordingly, without this improved standard FEM, the speed-up factor would be $>10$ instead of $>5$. For a comparison of standard FEM performance in comparison to other methods for solving Maxwell's equations we refer to previous benchmarks 16 18

In general, the Schur method speed-up factor obtained for a specific setup is expected to increase with the ratio of the sizes of the matrix blocks $A_{11}$ and $A_{22}$ (Eq. (2)). However, inverting the Schur complement requires 


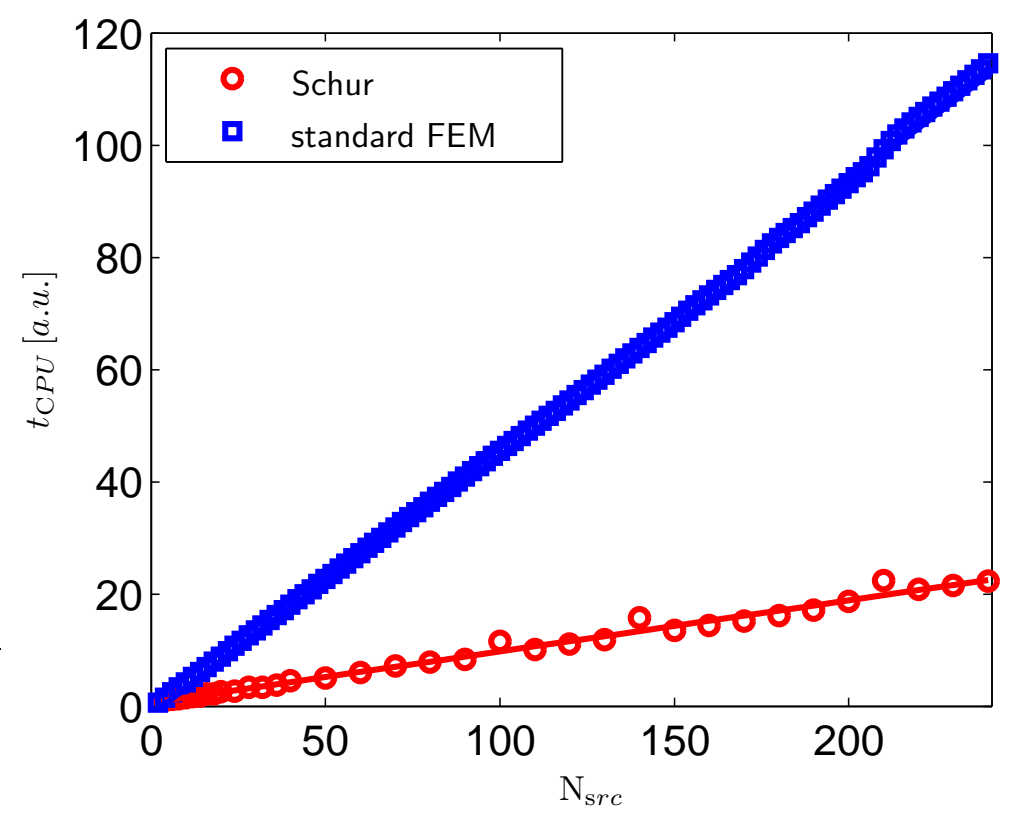

Figure 4. Comparison of computation times for simulations of the same setup using the Schur complement method (red circles, linear interpolation: red line) and using standard FEM (blue squares, linear interpolation: blue line): Computation time $t_{\mathrm{C} P U}$ as function of number of sources $\mathrm{N}_{\mathrm{s} r c}$.

inversion of a full matrix, such that the speed-up factor can be worsened for large problem sizes. Clearly, the $N$ computations for the standard ("no Schur") simulations can also be performed in parallel on a multi-core processor, thus decreasing computation time by a factor of $1 / N$. However, in typical applications where many spectra have to be computed, the computations using Schur complements can be parallelized in the same manner.

\section{CONCLUSION}

A finite-element method for computing solutions to the Maxwell 3D scattering problem has been presented, which makes use of a Schur complement solver for efficiently handling setups with many independent source terms for the same geometrical setup. A speed-up factor of greater than five has been observed for a specific application example in which angle-resolved reflection spectra from an array of silicon nano-posts are computed. We expect that this method can be used effectively for a variety of applications including computational metrology, computational lithography, nano-optics design in integrated optoelectronics, photovoltaics, light-emitters, and other fields.

\section{Acknowledgments}

We acknowledge support of BMBF through projects 13N13164 (SolarNano) and 13N12438 (MOSAIC).

\section{REFERENCES}

1. L. Pang, D. Peng, P. Hu, D. Chen, L. He, Y. Li, M. Satake, and V. Tolani, "Computational metrology and inspection (CMI) in mask inspection, metrology, review, and repair," Adv. Opt. Techn. 1, p. 299, 2012.

2. A. K. Wong, Optical Imaging in Projection Microlithography, SPIE Press, Bellingham, 2005.

3. P. Monk, Finite Element Methods for Maxwell's Equations, Oxford University Press, 2003.

4. K. Lai, "Review of computational lithography modeling: focusing on extending optical lithography and design-technology co-optimization," Adv. Opt. Techn. 1, p. 249, 2012.

5. G. Golub and C. Van Loan, Matrix Computations, Johns Hopkins University Press, 2012. 
6. J. Pomplun, S. Burger, L. Zschiedrich, and F. Schmidt, "Adaptive finite element method for simulation of optical nano structures," phys. stat. sol. (b) 244, p. 3419, 2007.

7. A. Schädle, L. Zschiedrich, S. Burger, R. Klose, and F. Schmidt, "Domain decomposition method for Maxwell's equations: Scattering off periodic structures," J. Comp. Phys. 226, p. 447, 2007.

8. S. Burger, L. Zschiedrich, J. Pomplun, S. Herrmann, and F. Schmidt, "Hp-finite element method for simulating light scattering from complex 3D structures," Proc. SPIE 9424, p. 94240Z, 2015.

9. J. Pomplun and F. Schmidt, "Accelerated a posteriori error estimation for the reduced basis method with application to 3D electromagnetic scattering problems," SIAM Journal on Scientific Computing 32, p. 498, 2010 .

10. F. Scholze, C. Laubis, G. Ulm, U. Dersch, J. Pomplun, S. Burger, and F. Schmidt, "Evaluation of EUV scatterometry for CD characterization of EUV masks using rigorous FEM-simulation," Proc. SPIE 6921, p. 69213R, 2008.

11. J. Pomplun, L. Zschiedrich, S. Burger, F. Schmidt, J. Tyminski, D. Flagello, and N. Toshiharu, "Reduced basis method for source mask optimization," Proc. SPIE 7823, p. 78230E, 2010.

12. B. H. Kleemann, J. Kurz, J. Hetzler, J. Pomplun, S. Burger, L. Zschiedrich, and F. Schmidt, "Fast online inverse scattering with Reduced Basis Method (RBM) for a 3D phase grating with specific line roughness," Proc. SPIE 8083, p. 808309, 2011.

13. P. Petrik, N. Kumar, M. Fried, B. Fodor, G. Juhasz, S. Pereira, S. Burger, and H. P. Urbach, "Fourier ellipsometry, an ellipsometric approach to fourier scatterometry," J. Europ. Opt. Soc. - Rap. 10, p. 15002, 2015.

14. S. Burger, L. Zschiedrich, J. Pomplun, M. Blome, and F. Schmidt, "Advanced finite-element methods for design and analysis of nano-optical structures: Applications," Proc. SPIE 8642, p. 864205, 2013.

15. E. D. Palik, Handbook of optical constants of solids, Academic press, 1985.

16. B. Maes, J. Petráček, S. Burger, P. Kwiecien, J. Luksch, and I. Richter, "Simulations of high-Q optical nanocavities with a gradual 1D bandgap," Opt. Express 21, p. 6794, 2013.

17. J. Hoffmann, C. Hafner, P. Leidenberger, J. Hesselbarth, and S. Burger, "Comparison of electromagnetic field solvers for the 3D analysis of plasmonic nano antennas," Proc. SPIE 7390, p. 73900J, 2009.

18. S. Burger, R. Köhle, L. Zschiedrich, W. Gao, F. Schmidt, R. März, and C. Nölscher, "Benchmark of FEM, waveguide and FDTD algorithms for rigorous mask simulation," Proc. SPIE 5992, p. 599216, 2005. 